\title{
Laser shock experiments to investigate and to model various aspects of the response of metals to shock loading
}

\author{
T. de Rességuier ${ }^{1, a}$, E. Lescoute ${ }^{1}$, L. Signor ${ }^{1}$, D. Loison ${ }^{1}$, A. Dragon ${ }^{1}$, M. Boustie ${ }^{1}$, \\ J.P. Cuq-Lelandais ${ }^{1}$, and L. Berthe ${ }^{2}$ \\ 1 Institut P' (UPR 3346), CNRS, ENSMA, Université de Poitiers, 1 Av. Clément Ader, \\ 86961 Futuroscope Cedex, France \\ 2 Laboratoire d'Application des Lasers de Puissance, PIMM, Arts et Métiers, ParisTech, France
}

\begin{abstract}
Laser driven shocks allow studying the dynamic behaviour of condensed matter over small spatial ( $\sim \mu \mathrm{m}$ to mm-order) and temporal ( $\sim \mathrm{ps}$ to ns-order) scales, at extremely high strain rates $\left(\sim 10^{7} \mathrm{~s}^{-1}\right)$. They can be used to test the predictive capability of constitutive models over wide ranges of loading pressures and pulse durations. We present experimental results in laser shock-loaded metals (iron, gold, tin), based on various, complementary techniques including time-resolved velocity measurements, transverse shadowgraphy and post-shock analyses of recovered samples. The data are used to investigate several shock wave processes such as yielding and polymorphic transformations, melting, spall fracture and dynamic fragmentation in both solid and melted states. On the basis of comparisons with numerical simulations, the abilities and limitations of several models are briefly discussed.
\end{abstract}

\section{Introduction}

All processes governing the response of condensed matter to shock loading, from elastic-plastic behaviour to phase transformations and dynamic fracture, are strain rate dependent to some extent. Explosives and plate impacts are widely used to study such shock wave processes at strain rates of about $10^{4}-10^{5} \mathrm{~s}^{-1}$ under pulsed pressure loads of a few $\mu \mathrm{s}$. Laser driven shocks can be used to extend these investigations over ranges of faster loading, typically some $10^{7} \mathrm{~s}^{-1}$, and shorter pulse durations of a few ns or less.

Here, we illustrate the use of laser shocks to investigate various aspects of materials dynamic behaviour at very high strain rates, and we test the predictive capability of numerical models under such uncommon loading conditions. In Section 2, the complex elastic-plastic response of iron, governed by both twinning and dislocation slip, and the well known polymorphic transition from $\alpha$ to $\varepsilon$ phase are studied over ranges of unusually short propagation distances. Section 3 concerns spall fracture and subsequent fragment ejection from laser shock-loaded iron and gold samples, then the evolution of this spallation process after shock-induced melting is explored, tin being chosen as a reference material because of its relatively low melting curve.

All experiments have been performed in the Laboratoire pour l'Utilisation des Lasers Intenses (LULI, UMR 7605, Ecole Polytechnique, France) or in the Centre d'Etudes Scientifiques et Techniques d'Aquitaine (CEA, Le Barp, France). The setup, fully described elsewhere [1-3], is schematically depicted in Fig. 1. The velocity of the sample free surface (opposite to the loaded surface) is measured either with a Velocity Interferometer System for Any Reflector (VISAR) or with a Photonic Doppler

\footnotetext{
a e-mail: resseguier@ensma.fr
}

This is an Open Access article distributed under the terms of the Creative Commons Attribution-Noncommercial License 3.0, which permits unrestricted use, distribution, and reproduction in any noncommercial medium, provided the original work is properly cited. 


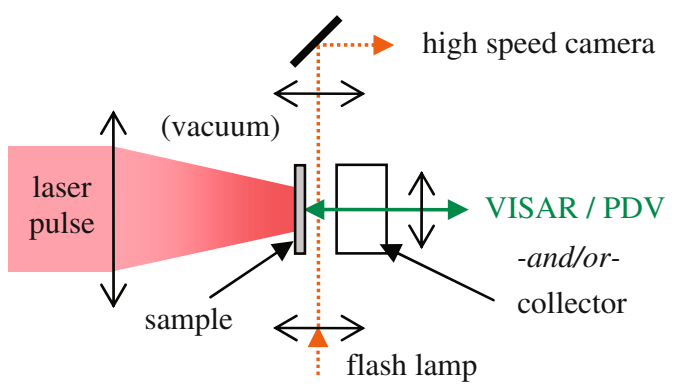

Fig. 1. Schematic of the experimental setup.

Velocimeter (PDV). Optical transverse shadowgraphy provides quasi-instantaneous pictures of the fragments ejected from this free surface. In some shots, ejecta are recovered on a plastic shield or in a gel collector [3] set a few mm away.

\section{Twinning and polymorphic transformations}

Owing to its importance in geophysics (as main constituent of the Earth's core) and in engineering applications, iron has been widely studied under both static and dynamic pressure. Still, open questions remain on wave propagation in this metal and on the kinetics of its structural changes.

Dynamic yielding of iron has been the subject of extensive studies for many years. A compression front splits into an elastic precursor followed by a slower plastic wave. The amplitude of the elastic precursor is reported to decrease with increasing propagation path length, and stress relaxation is observed before the plastic wave [4]. This highly rate-dependent behaviour has been attributed to the nucleation and motion of dislocations and deformation twinning [5]. Free surface velocity profiles measured in laser shock-loaded iron samples are plotted in Fig. 2, showing both elastic precursor decay and stress relaxation. Similar profiles have been recorded with piezoelectric transducers and reported in the past, together with micrographs of the recovered targets showing considerable twin formation [6]. The measured values of elastic particle velocities are much higher than those reported in thicker specimens under conventional shock loading [4,5], which confirms the rate-dependency of the response. A constitutive model [5] has been adapted to simulate this behaviour, as fully detailed elsewhere [6]. It involves a volume fraction of twinned material $\xi$ :

$$
\xi=\left[\xi_{0}^{1 / m}+\frac{1}{\theta} \int_{0}^{t} v_{t}(\tau) \mathrm{d} t\right]^{m}
$$

where $\xi_{0}$ is the initial fraction of twins, assumed to be zero, $\theta$ is a constant characteristic time, $m$ is set to 3 assuming 3D growth of twin platelets in thickness as well as in area, $\tau$ is the shear stress, and $v_{t}$ is a growth rate increasing linearly with stress above a threshold value $\tau_{0}$. Dislocation slip, assumed to be much faster than twinning, is treated as rate-independent through instantaneous accommodation of the plastic strain above a threshold stress $\tau_{1}$. The values of the model parameters $\theta, \tau_{0}$ and $\tau_{1}$ in laser shock-loaded iron have been determined as $18 \mathrm{~ns}, 0.4 \mathrm{GPa}$ and $1 \mathrm{GPa}$, respectively [6], significantly different from the original values fitted at lower strain rates. A fair agreement is obtained between measured and computed acceleration fronts (Fig. 2). Discrepancies after the deceleration from the peak velocity do not directly question the validity of the elastic-plastic model above, since this later part of the profiles is related to spall damage, as discussed further.

Another specificity of iron behaviour is the transformation from body-centered-cubic $(\alpha)$ to hexagonal-close-packed $(\varepsilon)$ structure above $13 \mathrm{GPa}$. Laser shocks allow investigating the kinetics of this transition because they involve times of pressure application of the same order as its relaxation time, namely a few ns to some tens of ns, according to data reported under conventional shock loading [4]. Figure 3 shows free surface velocity records in iron samples subjected to laser shocks of 


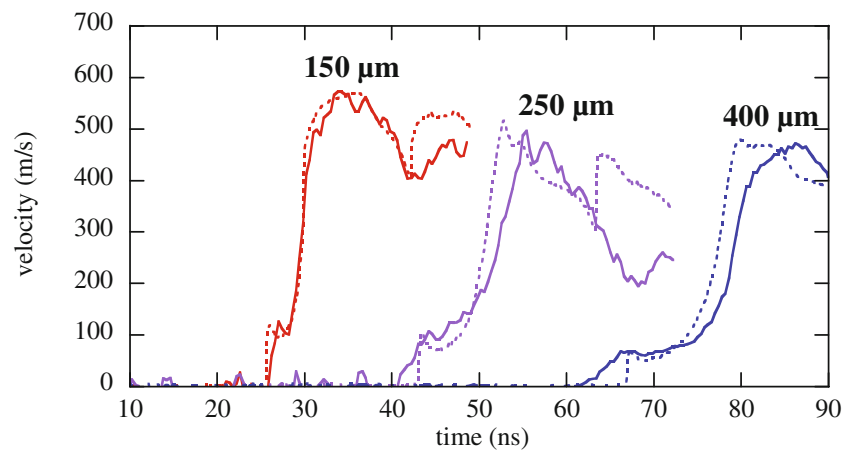

Fig. 2. Comparison between the free surface velocity profiles recorded (solid lines) and computed (dotted lines) in laser shock-loaded iron samples of thicknesses 150,250 and $400 \mu \mathrm{m}$.

(a)

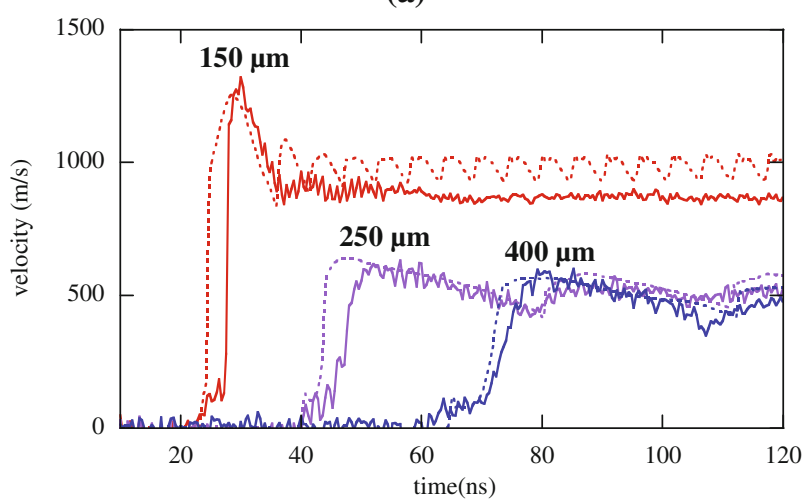

(b)

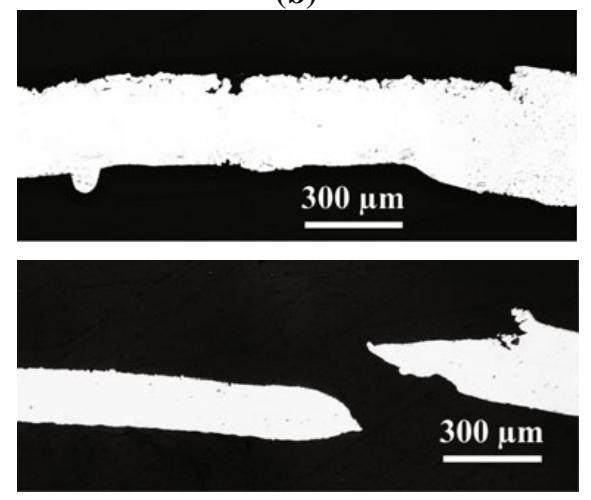

Fig. 3. Comparison between the free surface velocity profiles recorded (solid lines) and computed (dotted lines) in iron samples subjected to $\sim 130 \mathrm{GPa}$ laser shocks (a), and micrographs of cross sections in recovered samples (b), discussed in Section 3.

about $130 \mathrm{GPa}$. They are compared to the predictions of a model detailed elsewhere [7], involving a two-phase equation of state and a phenomenological expression of the transformation rate

$$
\frac{\mathrm{d} \alpha}{\mathrm{d} t}=\frac{M}{R T} \frac{\left(G_{\alpha}-G_{\varepsilon}\right)}{\eta}
$$

where $\alpha$ is the volume proportion of $\alpha$ phase, $M$ is the atomic mass of iron, $R$ is the perfect gas constant, $T$ is the temperature, $G_{\alpha}$ and $G_{\varepsilon}$ are the Gibbs free energies of both phases, and $\eta$ is a constant relaxation time characteristic of the transition kinetics. According to this model, the thinnest sample should be fully transformed to $\varepsilon$ phase throughout its $150 \mu \mathrm{m}$-thickness, while the two thicker ones should be only partially transformed (i.e. the region beneath the free surface should remain in the $\alpha$ phase, due to pressure decay below the $13 \mathrm{GPa}$ threshold). This leads to drastic differences in the wave profiles, discussed in detail in [2]. The above model is shown to provide consistent velocity profiles with a time constant $\eta$ set to $5 \mathrm{~ns}$ (Fig. 3), while shorter or longer values would lead to much poorer agreement, which gives some insight into the transformation kinetics.

\section{Spall fracture, dynamic fragmentation and "microspall"}

Dynamic failure, and more specifically spall fracture, which occurs upon tensile loading due to the interaction of incident and reflected release waves inside a shock-loaded sample, have been widely 

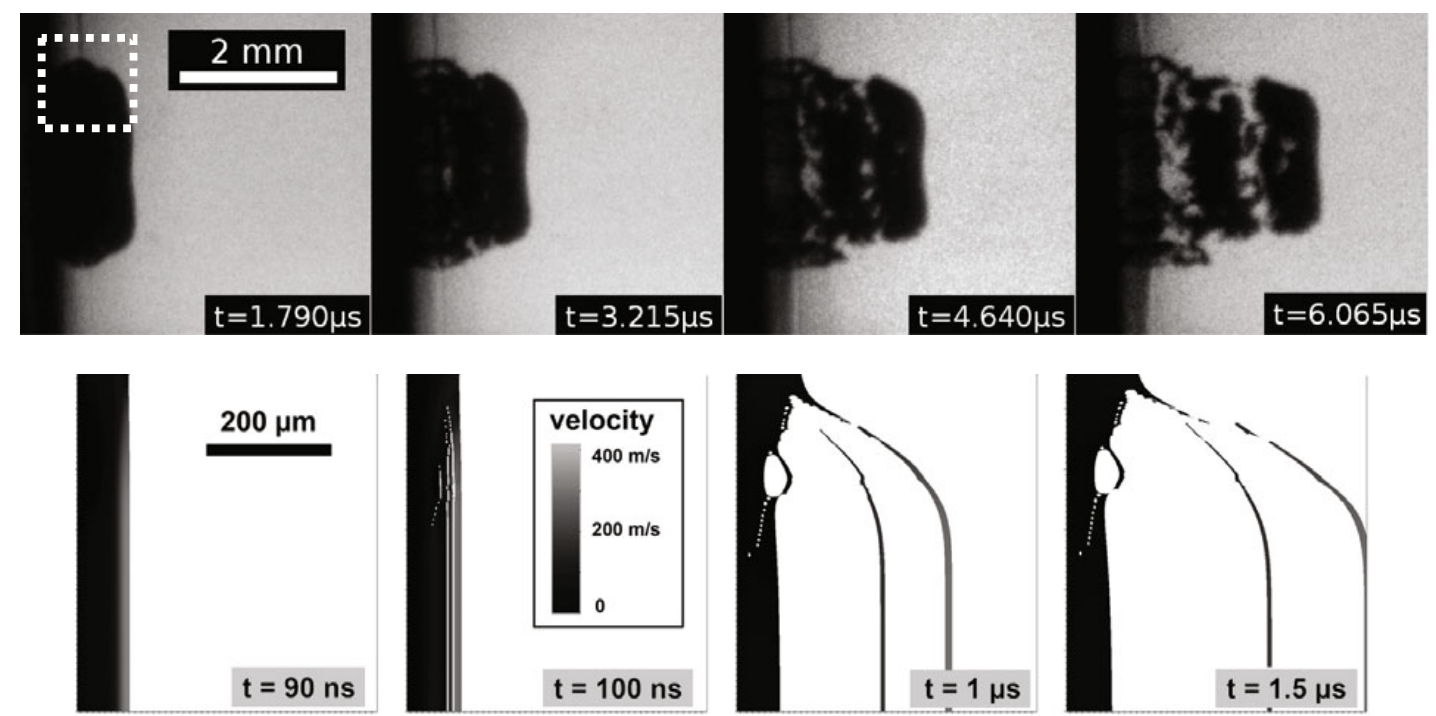

Fig. 4. Transverse shadowgraphs (top) showing the ejection (from left to right) of successive spalled layers from the free surface of a $300 \mu \mathrm{m}$-thick gold sample subjected to a $47 \mathrm{GPa}$ laser shock (shot at $t=0$ ), and simulation of this experiment (bottom), showing the early stages of this multiple spallation process, in a region corresponding to the white dotted line in the first frame (upper left picture).

studied for several decades, both theoretically and experimentally, for both basic and applied motivations [8]. Laser shocks can be used to extend such research toward extremely fast strain rates, where the measured tensile strength might approach its 'ideal' theoretical value [9]. Besides, their relatively low destructive character is a major advantage for post-test analyses of damage in recovered samples, even in brittle specimens, or for collecting the ejected fragments.

Thus, the results in Fig. 3 provide key information on the effects of the $\alpha-\varepsilon$ transition on spall fracture in iron. In the free surface velocity profiles, the amplitude of the so-called "pullback" from the peak value to the reacceleration indicative of spall damage is roughly proportional to the dynamic tensile strength of the material [8]. It is shown to be higher in the thinnest sample, where tension near the free surface is induced after the $\alpha-\varepsilon-\alpha$ cycle. This increase of the tensile strength after the phase change is accounted for in the model. Besides, the transformation affects the fracture surface morphology, as evidenced in the cross sections on the right, with a very clear change from a rough surface (top) to a "smooth spall" (bottom) already observed under conventional shock loading.

Much effort is devoted to characterizing and modelling the early stages of spall fracture (i.e. nucleation and growth of voids or micro-cracks), while the later ejection of fragments and their ballistic properties are a major concern in many practical applications. Figure 4 shows transverse shadowgraphs recorded behind a laser shock-loaded gold target (top), together with a two-dimensional simulation of this experiment (bottom). Fracture is modelled with a simple erosion criterion assuming instantaneous element deletion as soon as local tension reaches a constant strength, set to $4.1 \mathrm{GPa}$ on the basis of VISAR records of the free surface velocity, not shown here for brevity. Two distinct spalled layers are ejected successively, with significant curvature at their edges, and secondary fragments of various sizes are generated from the periphery of the loaded zone. A correct overall consistency is found between observations and calculations, including a very good match for the ejection velocities.

Little data can be found yet about how spall fracture and dynamic fragmentation evolve in a specimen fully or partially melted, on compression or on release, before wave interactions induce tensile loading. Upon reflection of a triangular compressive pulse (or "unsupported shock wave") from the free surface of a molten sample, cavitation is expected to occur under very low tension, very close to the free surface, and thus to lead to the ejection of a cloud of tiny droplets (referred to as "micro-spall") with a wide range of ejection velocities [10]. 
(a)

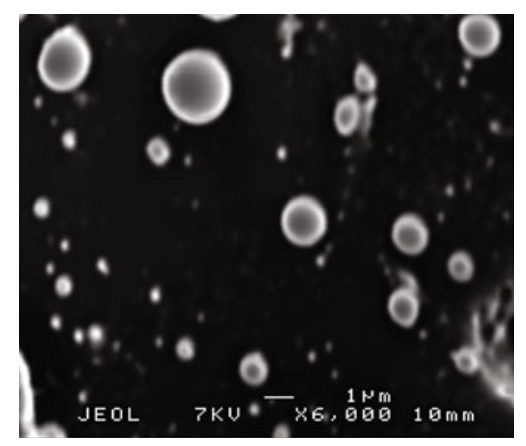

(b)

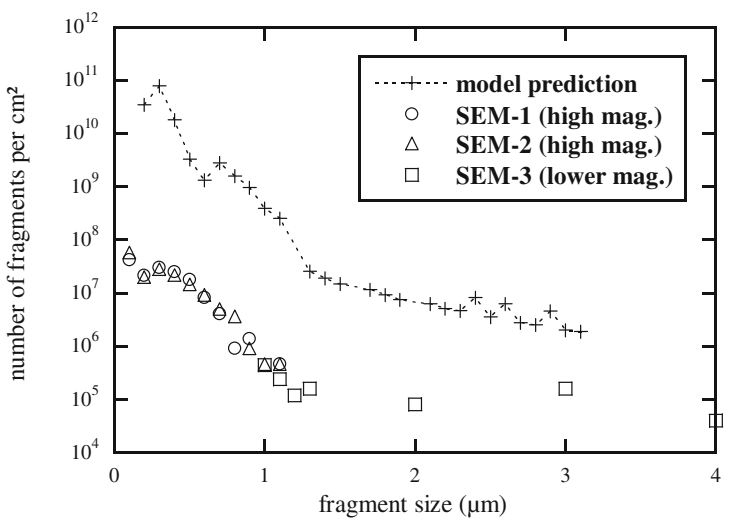

Fig. 5. Scanning Electron Micrograph (SEM) showing tin droplets recovered on the surface of a polycarbonate shield after being ejected from a $50 \mu$ m-thick sample subjected to a $140 \mathrm{GPa}$ laser shock (a), and comparison between the fragment size distributions inferred from such micrographs and that predicted by a simulation of this experiment using an energy-based fragmentation model (b).

High pressure laser shocks have been applied onto tin samples to quantify the progressive loss of tensile strength associated with incipient melting on release [11], to investigate the debris ejection from relatively thick specimens after shock-induced melting $[1,12]$, and to characterize more specifically the micro-spall ejected from thin foils [13]. In parallel, some theoretical issues have been addressed [14,15] and a well known, energy-based fragmentation model from the literature [16] has been adapted to the case of liquid metals then implemented in a hydrocode. Figure 5 illustrates a comparison between the predictions of this model and the experimental fragment size distributions inferred from image analyses performed on scanning electron micrographs of a plastic shield impacted by tin droplets ejected from a $50 \mu \mathrm{m}$-thick foil subjected to a $140 \mathrm{GPa}$ laser shock. The spherical shape of the particles, due to surface tension, indicate that they were generated in the liquid state, after shockinduced melting, then at least partially resolidified before impacting the shield. A good consistency is obtained on the fragment sizes, ranging from about 0.2 to $3 \mu \mathrm{m}$, with a mean value of about $0.6 \mu \mathrm{m}$. However, the calculation predicts fragment numbers per unit area much greater than observed experimentally (see the vertical shift in the graph). The major cause for this discrepancy is probably the penetration of a large number of tin droplets below the impacted surface of the shield. Since smaller fragments should be ejected at higher velocities and have less drag during penetration, they should imbed more easily into the polycarbonate, so that the discrepancy between theoretical and observed fragment numbers decreases with increasing fragment size.

Current work on this subject includes 3D micro-tomography to characterize the whole debris population recovered within "soft" collectors of low density (foams and gels), analogous work on other metals, of engineering or geophysical interest, and development of a physically-based model involving a more accurate description of shock-induced melting.

\section{Conclusion}

The main specificity of laser driven shocks is the very short time of pressure application, typically a few $10^{-9} \mathrm{~s}$ or less. On one hand, this makes direct measurements within the shock-compressed matter challenging, because they require a very high time resolution. Besides, this short duration implies fast pressure decay during propagation from the loaded surface into the sample, which has to be carefully characterized and taken into account in the interpretations of the results. On the other hand, such short pulses allow studying the dynamic behaviour of materials over ranges of extremely high strain rates and very small propagation distances, where the kinetics of the various processes governing this behaviour play key roles, non-equilibrium states can be investigated, and metastable structures may 


\section{EPJ Web of Conferences}

be quenched. Finally, due to this short duration, the overall deformation and motion of a laser shockloaded target are usually small, so that both the damaged specimen and the ejected fragments can be partially recovered for post-test analyses that can be very informative. Thus, laser shock loading appears as a useful complement to more conventional experimental techniques, to test the ability of existing models to describe the dynamic response of condensed matter (as has been illustrated in the present paper), and to develop new ones with improved predictive capabilities.

\section{References}

1. T. de Rességuier, L. Signor, A. Dragon, M. Boustie, L. Berthe, Appl. Phys. Lett. 92, 131910 (2008)

2. T. de Rességuier, M. Hallouin, Phys. Rev. B 77, 174107 (2008)

3. E. Lescoute, T. de Rességuier, J.M. Chevalier, M. Boustie, J.P. Cuq-Lelandais, L. Berthe, Appl. Phys. Lett. 95, 211908 (2009)

4. L.M. Barker, R.E. Hollenbach, J. Appl. Phys. 45, 4872 (1974)

5. J.N. Johnson, R.W. Rhode, J. Appl. Phys. 42, 4171 (1971)

6. T. de Rességuier, M. Hallouin, J. Appl. Phys. 84, 1932 (1998)

7. T. de Rességuier, M. Hallouin, J. Appl. Phys. 90, 4377 (2001)

8. T. Antoun, L. Seaman, D.R. Curran, G.I. Kanel, S.V. Razorenov, A.V. Utkin, Spall Fracture (Springer, New York, 2002)

9. G.I. Kanel, Int. J. Frac. 163, p. 173 (2010)

10. P. Andriot, P. Chapron, V. Lambert, F. Olive, Shock Waves in Condensed Matter, p. 277 (1983)

11. T. de Rességuier, L. Signor, A. Dragon, P. Severin, M. Boustie, J. Appl. Phys. 102, 073535 (2007)

12. T. de Rességuier, L. Signor, A. Dragon, M. Boustie, G. Roy, F. Llorca, J. Appl. Phys. 101, 013506 (2007)

13. T. de Rességuier, L. Signor, A. Dragon, G. Roy, Int. J. Frac. 163, p. 109 (2010)

14. L. Signor, A. Dragon, G. Roy, T. de Rességuier, F. Llorca, Arch. Mech. 60, p. 323 (2008)

15. L. Signor, T. de Rességuier, A. Dragon, G. Roy, A. Fanget, M. Faessel, Int. J. Imp. Eng. 37, p. 887 (2010)

16. D.E. Grady, J. Mech. Phys. Solids 36, p. 353 (1988) 